\title{
Prediction of Composite Laminate Strength Properties Using a Refined Zigzag Plate Element
}

\author{
A. Barut ${ }^{1}$, E. Madenci ${ }^{2}$ \\ University of Arizona, Tucson, AZ, 85721, USA \\ and \\ A. Tessler ${ }^{3}$, \\ NASA Langley Research Center, Hampton, VA 23681-2199, USA
}

\begin{abstract}
This study presents an approach that uses the refined zigzag element, $\operatorname{RZE}^{\{2,2\}}$ in conjunction with progressive failure criteria to predict the ultimate strength of composite laminates based on only ply-level strength properties. The methodology involves four major steps: (1) Determination of accurate stress and strain fields under complex loading conditions using $\mathrm{RZ} \mathrm{E}^{\{2,2\}}$-based finite element analysis, (2) Determination of failure locations and failure modes using the commonly accepted Hashin's failure criteria, (3) Recursive degradation of the material stiffness, and (4) Non-linear incremental finite element analysis to obtain stress redistribution until global failure. The validity of this approach is established by considering the published test data and predictions for (1) strength of laminates under various off-axis loading, (2) strength of laminates with a hole under compression, and (3) strength of laminates with a hole under tension.
\end{abstract}

\section{Introduction}

Laminated fiber-reinforced polymer (FRP) composites exhibit directional stiffness and strength properties, and offer different fabrication architecture; thus, enabling advanced design concepts, structural tailoring, multifunctional features, and performance enhancements. Their actual applications, however, are often lagging behind the material development and fabrication because characterization of their mechanical and strength properties requires large amounts of data to achieve an adequate confidence level. The ability to model and simulate composite materials for strength prediction based on ply level material property data can reduce the time and cost for material testing and characterization; thus, provide the opportunity to use advanced material systems on critical military and commercial equipment in a timely manner.

Strength of FRP composite laminates depends on fiber directions, type of fibers and resins, number of layers, stacking sequence, type of loading, environmental conditions, and random variation of material and strength properties due to manufacturing. Their failure involves a progressive series of events with discrete failure modes such as matrix cracking, fiber-matrix shear, fiber breakage, and delamination. The presence of such failure modes results in stiffness degradation. This leads to stress redistribution in the layers and constituents. Therefore, to enable realistic simulation of composite-laminate strength, accurate predictions of stress redistribution is paramount.

The stress state in a composite laminate is dependent on the loading conditions, fiber- and resin-rich layer thickness, face-sheet lay-up, core thickness and process-dependent properties. Understanding the behavior of such structures can result in weight and cost savings by designing against unnecessary conservatism. Finite element Analysis (FEA) is widely utilized to assess the strength of such structures. To model a vast majority of the potential

\footnotetext{
${ }^{1}$ Research Associate Professor, Department of Aerospace and Mechanical Engineering, Member AIAA.

${ }^{2}$ Professor, Department of Aerospace and Mechanical Engineering, Senior Member AIAA.

${ }^{3}$ Senior Research Scientist, Structural Mechanics \& Concepts Br., Research \& Technology Directorate.
} 
failure modes, however, an extremely detailed finite element discretization is often required. Using traditional elements, through-the-thickness finite element discretizations are often impractical. This is because an extremely high-density mesh is required to maintain a proper aspect ratio between the elements in the fiber- and resin-rich layers. Typically, composite laminates have a low ratio of thickness to in-plane characteristic dimension; thus, they can be modeled using 2-D plate elements that are based on an assumed set of kinematical relations. However, they require shear correction in order improve transverse shear deformation. The constitutive relations are in terms of average stress resultant quantities rather than point stresses and strains. Strength prediction requires an accurate 3-D stress field especially if edge delamination is the source of progressive failure.

Material characterization of laminated composite structures is well established, and failure prediction models for damage modes associated with FRP composite laminates are relatively successful. Ochoa and Reddy [6] present an excellent discussion of progressive failure analyses. In laminated composite structures, fiber-dominated failure modes (e.g., axial tension) are better-understood and analyzed than matrix-dominated failure modes (e.g., axial compression, transverse tension). To predict ultimate strength of a laminate, there exist two commonly accepted methods: damage mechanics and progressive ply failure. The damage-mechanics approach employs physically based equations for damage initiation and evolution while taking into account the material's microstructure [1-3]. However, it requires extensive material characterization for damage parameters, and in most cases such measurements are not feasible. Progressive ply failure combines failure criteria for damage mode identification [45] and degradation [6-9] of the material stiffness. The value of degradation factor is not-physically based; it is assigned a small enough value in order to ensure the convergence of finite element analysis. Methods to predict failure initiation and to perform material degradation remain active areas of research. Material degradation can be performed using a ply-discounting approach or an internal state variable approach based on continuum damage mechanics. Most composite failure analysis methods embedded within a finite element analysis tool perform a point-stress analysis, evaluate failure criteria, possibly degrade material properties, and then continue to the next solution increment.

There exist many progressive failure models in the literature to predict the strength of FRP composite materials. These previous models utilize ply level strength parameters; however, they lack reliability in their strength predictions under longitudinal and transverse tension and compression, longitudinal and transverse open-hole tension and open-hole compression. The lack of reliability is associated with inaccurate computation of stress redistribution within the conventional finite elements used to model composite laminates. The existing conventional elements based on the first order shear deformation theory (FSDT) require different shear correction factors in the presence of a degraded ply or plies through the thickness, and fail to provide accurate and complete stress field near the free edges.

To evaluate the strength of a composite structure with a high degree of fidelity, it is first essential to predict accurate stress and strain fields, and then utilize a computationally efficient and accurate progressive damage model. For these reasons, an accurate and robust finite element analysis is required that can account for the discrete nature of both the fiber- and resin- rich layers of the individual plies, as well as the variation of stiffness and strength properties of the core. Recently, a viable theoretical framework called the Refined Zigzag Theory (RZT) was developed by Tessler et al. [10-13]. The theory uses a fixed number of kinematic variables (seven for plate analysis) and is well suited for the analysis of a wide range of composite and sandwich laminates without the use of shear correction factors. Efficient RZT-based finite element formulations for the analysis of beam and plate structures were also developed [14-16]. Focusing specifically on higher-order deformation effects commonly associated with thick-section laminated composite and sandwich panels, Barut et al. [17] explored a higher-order kinematic representation using RZT as its basis. In their higher-order RZT formulation, Barut et al. used the same zigzag functions as those of RZT; however, higher-order in-plane deformation modes were added using a set of piecewisequadratic zigzag contributions and, moreover, both linear and quadratic polynomial expansions were added for the transverse displacement component. Hence, this higher-order RZT is defined by the notation RZT $^{\{2,2\}}$, in which the first and second superscripts denote the order of expansions used for the in-plane and transverse displacement components, respectively.

The focus of this study is to present a validated approach based on the refined zigzag element, $\mathrm{RZE}^{\{2,2\}}$ by Barut et al. [17] used in conjunction with the progressive failure criteria of Hashin [4] to predict the ultimate strength of composite laminates utilizing the ply-level strength properties only. The validity of this approach is 
established by considering the published test data and predictions for (1) strength of laminates under various off-axis loading [18, 19], (2) strength of laminates with a hole under compression [20], and (3) strength of laminate with a hole under tension, [8, 21-22].

\section{Approach}

This approach involves four major steps: (1) determination of accurate stress and strain fields under complex loading conditions using $\mathrm{RZE}^{\{2,2\}}$ based finite element analysis, (2) determination of failure site(s) and modes using commonly accepted failure criteria of Hashin, (3) recursive degradation of the material stiffness, and (4) non-linear incremental finite element analysis to obtain stress redistribution until global failure.

\section{Refined Zigzag Element, RZE ${ }^{\{2,2\}}[17]$}

This element offers substantial analytic and computational advantages for the analysis of homogeneous, laminated composite, and sandwich laminates. Starting from a multi-scale kinematic description represented by a superposition of the coarse and fine kinematics, it employs a parabolic transverse displacement assumption and higher-order RZT-based in-plane displacements to derive a higher-order refine zigzag plate theory for laminated composite and sandwich plates. The displacement assumptions involve eleven kinematic variables and are given as

$$
\begin{aligned}
& u_{1}^{(k)}(\mathbf{x}, z)=u(\mathbf{x})+z \theta_{1}(\mathbf{x})+\phi_{1}^{(k)}(z) \psi_{11}(\mathbf{x})+\frac{z}{h} \phi_{1}^{(k)}(z) \psi_{12}(\mathbf{x}) \\
& u_{2}^{(k)}(\mathbf{x}, z)=v(\mathbf{x})+z \theta_{2}(\mathbf{x})+\phi_{2}^{(k)}(z) \psi_{21}(\mathbf{x})+\frac{z}{h} \phi_{2}^{(k)}(z) \psi_{22}(\mathbf{x}) \\
& u_{3}(\mathbf{x}, z)=w(\mathbf{x})+\frac{z}{h} w_{1}(\mathbf{x})+\left(\frac{z^{2}}{h^{2}}-\frac{1}{3}\right) w_{2}(\mathbf{x})
\end{aligned}
$$

where $\mathbf{x} \equiv\left(x_{1}, x_{2}\right)$ are the in-plane coordinates, $z \in(-h, h)$ is the thickness coordinate, with $2 h$ denoting the total plate thickness; $\phi_{\alpha}^{(k)}(z)$ are the piecewise linear zigzag functions within the $k^{\text {th }}$ layer that were originally derived in [10]. The kinematic variables $u(\mathbf{x})$ and $v(\mathbf{x})$ are the through-the-thickness uniform components of the in-plane displacements in the $x$ - and $y$-directions, respectively. The kinematic variable $w(\mathbf{x})$ is an average transverse displacement defined in [17]. The additional transverse-displacement variables, $w_{1}(\mathbf{x})$ and $w_{2}(\mathbf{x})$, represent the symmetric and anti-symmetric thickness-stretch modes. The kinematic variables $\theta_{1}(\mathbf{x})$ and $\theta_{2}(\mathbf{x})$ denote the bending rotations about the negative $x$ and positive $y$ axes, respectively. The zigzag amplitudes defined by the variables $\psi_{\alpha 1}(\mathbf{x})$ and $\psi_{\alpha 2}(\mathbf{x})(\alpha=1,2)$ permit both non-symmetric, $\psi_{\alpha 1}(\mathbf{x})$, and symmetric, $\psi_{\alpha 2}(\mathbf{x})$, in-plane zigzag deformation modes. The contributions associated with $\psi_{12}(\mathbf{x}), \psi_{22}(\mathbf{x}), w_{1}(\mathbf{x})$, and $w_{2}(\mathbf{x})$ are the additional, higher-order terms that do not appear in the RZT model [10-13] . In addition, an average transverse normal stress, $\sigma_{z z}$, is independently assumed as a cubic function through the laminate thickness as

$$
\sigma_{z z}(\mathbf{x}, z)=\sigma_{z 0}(\mathbf{x})+\sigma_{z 1}(\mathbf{x})\left(\frac{z}{h}-\frac{z^{3}}{3 h^{3}}\right)
$$

where $\sigma_{z 0}(\mathbf{x})$ and $\sigma_{z 1}(\mathbf{x})$ are functions of the kinematic variables defining the displacement field in Eq. (1) (refer to [17] for the details of derivation of these functions.)

The zigzag kinematic assumptions are represented by piecewise-linear $\mathrm{C}^{0}$-continuous functions through the thickness. The zigzag kinematic framework enables sufficiently accurate and computationally efficient modeling of a wide range of homogeneous and heterogeneous laminates without the use of shear correction factors. Novel zigzag functions, derived a priori from constitutive relations without enforcing debilitating stress-equilibrium constraints, are responsible for overcoming several critical shortcomings of the earlier zigzag theories. The stress resultants obtained from the equilibrium equations are physically consistent with their definitions based on Hooke's relations. 
In addition to the in-plane deformations, this element includes both transverse shear and transverse normal (thickness-stretch) deformations, and computes accurately the in-plane and transverse stress components through the thickness, i.e., it permits the determination of all six stress and strain components. This $\mathrm{C}^{0}$-continuous element maintains a fixed number of kinematic unknowns regardless of the number of material layers. Using the principle of virtual work, equilibrium equations and consistent boundary conditions are derived in a variationally consistent manner.

As shown in Figure 2, the finite element is a six-node triangle consisting of three corner nodes and three midside nodes along the edges. At each corner node, eleven degrees of freedom (DOF) are specified. These are the inplane displacements, $u^{(i)}$ and $v^{(i)}$, the transverse displacements, $w^{(i)}, w_{1}^{(i)}$, and $w_{2}^{(i)}$, the bending rotations, $\theta_{1}^{(i)}$ and $\theta_{2}^{(i)}$, and the zigzag amplitudes (rotations), $\psi_{11}^{(i)}, \psi_{12}^{(i)}, \psi_{21}^{(i)}$, and $\psi_{22}^{(i)}$, with $i=1,2,3$ denoting the corner nodes. At the mid-side nodes, only the transverse displacements, $w^{(i+3)}, w_{1}^{(i+3)}$, and $w_{2}^{(i+3)}(i=1,2,3)$, are defined.

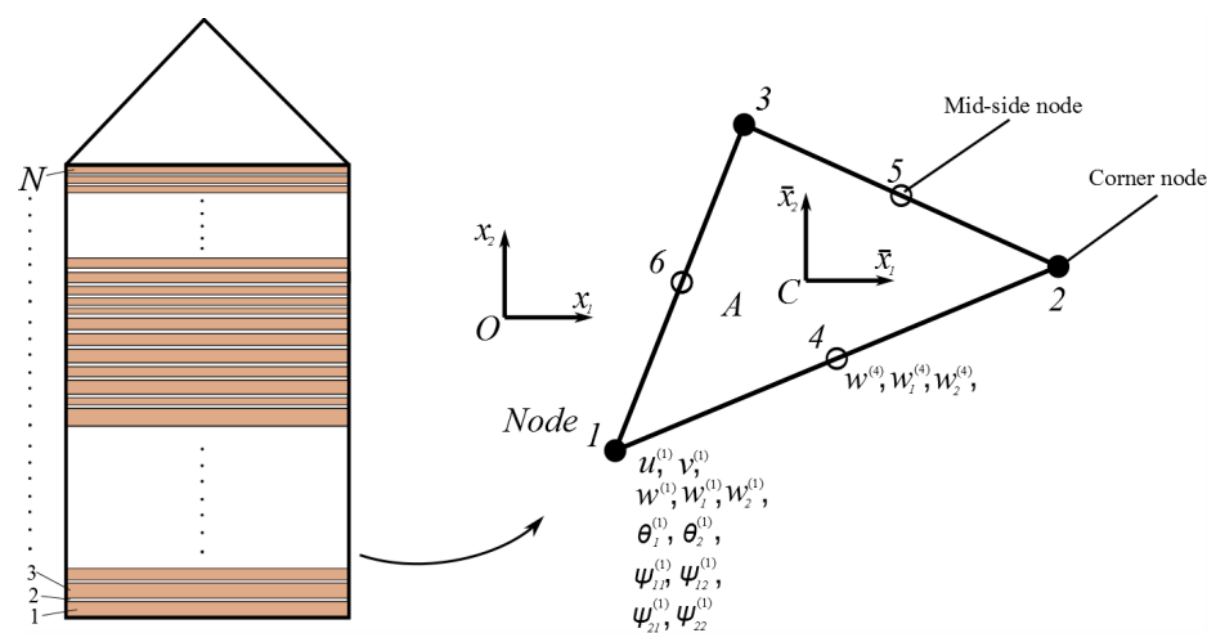

Fig. 2. Description of 6-node anisoparametric element with nodal DOF assignment

The non-homogeneous nature of a laminated composite is considered by including discrete fiber- and resin-rich layers. The element is capable of a highly detailed modeling of each ply (fiber- and resin-rich layers regardless of the number of plies) as well as the stiffness degradation of any of the layers. Unlike the existing finite elements for composite laminates, the present provides robust and accurate prediction of all six stress components (in-plane and transverse normal and shear stresses). The key features of this element are summarized as

- Employs refined zigzag theory

- Accounts for the through-thickness stretch and shear deformations

- Uses anisoparametric interpolations that do not suffer from shear locking

- Requires no shear correction factors to yield accurate predictions

- Uses zigzag functions that accurately model the layerwise shear stiffnesses

- Models accurately the fiber- and resin-rich regions, in addition to the highly stiff and very compliant layers

- Provides highly effective modeling for thick-section laminates, sandwich structures, and bonded joints

- Models accurately the in-plane, transverse normal, and transverse shear stresses

- Computes highly accurate interlaminar stresses via integration of equilibrium equations

\section{Progressive failure criteria}

Failure modes in laminated composite panels are strongly dependent on ply orientation, loading direction and panel geometry. There are four basic modes of failure that occur in a laminated composite structure. These failure modes are; matrix cracking, fiber-matrix shear failure, fiber failure, and delamination. To simulate damage growth accurately, the failure analysis must be able to predict the failure mode in each ply and apply the corresponding 
reduction in material stiffness. The failure criteria included in the present analyses are those proposed by Hashin [4] as

Matrix compressive failure --- $\left(\sigma_{22}+\sigma_{33}\right) \leq 0$

$\left(e_{m}^{c}\right)^{2}=\left[\left(\frac{Y_{c}}{2 S_{23}}\right)^{2}-1\right]\left(\frac{\sigma_{22}+\sigma_{33}}{Y_{c}}\right)+\frac{\left(\sigma_{22}+\sigma_{33}\right)^{2}}{4 S_{23}^{2}}+\frac{\left(\sigma_{23}^{2}-\sigma_{22} \sigma_{33}\right)}{S_{23}^{2}}+\left(\frac{\sigma_{12}}{S_{12}}\right)^{2}+\left(\frac{\sigma_{13}}{S_{13}}\right)^{2}= \begin{cases}>1 & \text { failure } \\ \leq 1 & \text { no failure }\end{cases}$

Matrix tensile failure --- $\left(\sigma_{22}+\sigma_{33}\right) \geq 0$

$\left(e_{m}^{t}\right)^{2}=\frac{\left(\sigma_{22}+\sigma_{33}\right)^{2}}{Y_{t}^{2}}+\frac{\left(\sigma_{23}^{2}-\sigma_{22} \sigma_{33}\right)}{S_{23}^{2}}+\left(\frac{\sigma_{13}}{S_{13}}\right)^{2}+\left(\frac{\sigma_{23}}{S_{23}}\right)^{2}= \begin{cases}>1 & \text { failure } \\ \leq 1 & \text { no failure }\end{cases}$

Fiber-matrix-shear compressive failure --- $\sigma_{11} \leq 0$

$\left(e_{s}^{c}\right)^{2}=\left(\frac{\sigma_{11}}{X_{c}}\right)^{2}+\left(\frac{\sigma_{12}}{S_{12}}\right)^{2}+\left(\frac{\sigma_{13}}{S_{13}}\right)^{2}= \begin{cases}>1 & \text { failure } \\ \leq 1 & \text { no failure }\end{cases}$

Fiber-matrix-shear tensile failure --- $\sigma_{11} \geq 0$

$\left(e_{s}^{t}\right)^{2}=\left(\frac{\sigma_{12}}{S_{12}}\right)^{2}+\left(\frac{\sigma_{13}}{S_{13}}\right)^{2}= \begin{cases}>1 & \text { failure } \\ \leq 1 & \text { no failure }\end{cases}$

Fiber compressive failure --- $\sigma_{11} \leq 0$

$\left(e_{1}^{c}\right)^{2}=\left(\frac{\sigma_{11}}{X_{c}}\right)^{2}= \begin{cases}>1 & \text { failure } \\ \leq 1 & \text { no failure }\end{cases}$

Fiber tensile failure $\sigma_{11} \geq 0$

$\left(e_{1}^{t}\right)^{2}=\left(\frac{\sigma_{11}}{X_{t}}\right)^{2}+\frac{\sigma_{12}^{2}+\sigma_{13}^{2}}{S_{12}^{2}}= \begin{cases}>1 & \text { failure } \\ \leq 1 & \text { no failure }\end{cases}$

Delamination

$e_{d}^{2}=\left(\frac{\sigma_{33}}{Y_{t}}\right)^{2}+\left(\frac{\sigma_{13}}{S_{13}}\right)^{2}+\left(\frac{\sigma_{23}}{S_{23}}\right)^{2}=\left\{\begin{array}{ll}>1 & \text { failure } \\ \leq 1 & \text { no failure }\end{array}\right.$ for $\sigma_{33} \geq 0$

in which $\sigma_{i j}$ with $(i, j=1,2,3)$ are the stress components in reference to the material coordinate system. The parameters $\left(X_{t}, X_{c}\right)$ and $\left(Y_{t}, Y_{c}\right)$ represent the tension and compression strengths along the fiber and transverse directions, respectively. The in-plane shear and transverse shear strengths are $S_{12}, S_{13}$ and $S_{23}$, respectively.

\section{Recursive degradation}

Degradation of ply stiffness is a common approach as part of the laminate failure analysis. Based on the stresses and strains in each ply, a lamina failure criterion such as that of Hashin [4] is employed to determine the particular ply which fails first and its failure mode. Typical values for the degradation factor can range from a very small value (e.g., 10-6) to a large value (e.g., 0.8) for this type of material degradation. As suggested by Knight [23], recursive degradation successively degrades the material stiffness coefficients in a gradual manner to avoid numerical difficulties arising from abrupt changes in material stiffness. When the degradation factor is not small (for example, 0.5), then some of the numerical convergence issues associated with an instantaneous local change in material stiffness are avoided. Specifying recursive degradation with a near-zero degradation factor is nearly equivalent to specifying instantaneous degradation with the same near-zero factor. 


\section{Non-linear incremental finite element analysis for stress redistribution}

The laminate with reduced stiffness is again analyzed for stresses and strains. The lamina failure criterion predicts the next ply failure, and laminate stiffness is accordingly reduced again. This cycle continues until ultimate laminate failure is reached using an incremental approach in accordance with the incremental equilibrium equation in the form

$$
\mathbf{K}\left(\mathbf{C}_{k}^{(t+\Delta t, i)}\right) \Delta \mathbf{u}^{(t+\Delta t, i+1)}=\mathbf{R}^{(t+\Delta t)}-\mathbf{f}^{(t+\Delta t, i)}
$$

in which $\mathbf{K}\left(\mathbf{C}_{k}^{(t+\Delta t, i)}\right)$ represents the tangent stiffness of $\mathrm{RZE}$ at load step $(t+\Delta t)$ in the $i^{t h}$ iteration with $\mathbf{C}_{k}^{(t+\Delta t, i)}=\mathbf{C}_{k}^{(t+\Delta t, i)}\left(E_{11}, E_{22}, E_{33}, G_{12}, G_{13}, G_{23}, v_{12}, v_{13}, v_{23}\right)$ being the material stiffness matrix of the $k^{t h}$ ply in laminate.

The known external force is represented by $\mathbf{R}^{(t+\Delta t)}$, and the internal force vector by $\mathbf{f}^{(t+\Delta t, i)}$. The unknown incremental displacement vector at the $(i+1)^{t h}$ is $\Delta \mathbf{u}^{(t+\Delta t, i+1)}$. Until first ply failure, the material stiffness for each ply remains as original and not degraded, i.e., $\mathbf{C}_{k}^{(t+\Delta t, i)}=\mathbf{C}_{k}^{(0)}$. At the onset of ply failure, the sets of ply stiffness properties, $\left(E_{11}, E_{22}, G_{12}, G_{23}, G_{13}, v_{12}\right),\left(E_{22}, v_{12}\right),\left(G_{12}, G_{13}, v_{12}\right)$, and $\left(E_{33}\right)$ for fiber failure, matrix failure, fibermatrix shear, delamination, respectively, are degraded by a factor of $\beta$ whose initial value is 0.5. After each load increment, these sets of ply properties are recursively degraded as follows

$$
\begin{aligned}
& E_{i j}^{(t)}=\beta_{E_{i j}}^{(t)} E_{i j}^{(0)} \text { with } \beta_{E_{i j}}^{(t)}=\beta \beta_{E_{i j}}^{(t-\Delta t)} \\
& G_{i j}^{(t)}=\beta_{G_{i j}}^{(t)} G_{i j}^{(0)} \text { with } \beta_{G_{i j}}^{(t)}=\beta \beta_{G_{i j}}^{(t-\Delta t)} \\
& v_{i j}^{(t)}=\beta_{v_{i j}}^{(t)} v_{i j}^{(0)} \text { with } \beta_{v_{i j}}^{(t)}=\beta \beta_{v_{i j}}^{(t-\Delta t)}
\end{aligned}
$$

in which $E_{i j}^{(0)}, G_{i j}^{(0)}$ and $v_{i j}^{(0)}$ represent the elastic moduli, shear moduli and Poisson's ratios, respectively, for an undamaged ply. The ultimate laminate failure is established when the determinant of tangent stiffness matrix becomes nearly zero.

\section{Numerical Results}

The numerical results demonstrate the capability of this approach by first comparing the present predictions with the laminate tensile experiments by Sun et al. [18] and the predictions by Triplett [19 ]. Previous experimental results by Suemasu et al [20] serve as a validation for a laminate with an open hole under compression. The experimental results by Chang and Chang [8] and previous predictions by Sleight [21], and Gunel and Kayral [22] are considered in the validation of predictions for a laminate with a hole under tension.

\section{Strength of laminates under off-axis loading}

The laminates that are considered are made of AS4/ 3501-6 Gr/Epoxy unidirectional plies; they have the plylevel properties summarized in Table 1 .

Table 1. Ply-level properties for AS4/3501-6 Gr/Ep system Sun et al. [18]

\begin{tabular}{|l|c|c|l|}
\hline$E_{11}$ & $153.7 \mathrm{GPa}$ & $X_{t}$ & $2171.0 \mathrm{MPa}$ \\
\hline$E_{22}$ & $11.0 \mathrm{GPa}$ & $X_{c}$ & $-2013.0 \mathrm{MPa}$ \\
\hline$G_{12}$ & $6.9 \mathrm{GPa}$ & $Y_{t}$ & $67.0 \mathrm{MPa}$ \\
\hline$v_{12}$ & 0.32 & $Y_{c}$ & $-206.8 \mathrm{MPa}$ \\
\hline$t_{p l y}$ & $0.13 \mathrm{~mm}$ & $S_{12}$ & $110.3 \mathrm{MPa}$ \\
\hline
\end{tabular}

As pointed out by Sun et al. [18], off-axis loading is achieved by rotating all plies of the laminate by the same amount as the angle of loading. The finite element model of each laminate is composed of two triangular elements 
(RZE), and the boundary and loading conditions are shown in Fig. 3. This configuration results in a uniform strain field. The predictions from the proposed approach are compared with the actual strength measured by Sun et al. [18] and other predictions by Triplett [19], in which only one quadrilateral element subjected to constant strain conditions is employed to determine both the first-ply and ultimate failure loads of a laminate; these are summarized in Tables 2-5 for various off-axis loading angles. As discussed by Sun et al. [18], the degree of agreement between the predicted and measured strength values depends on the off-axis angle. The agreement is remarkably good for the majority of the cases, and remains quite respectable for the remaining cases.

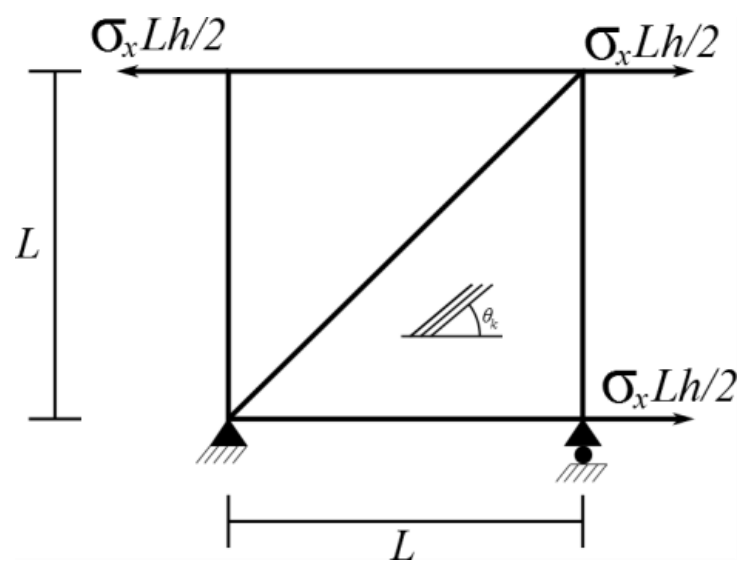

Fig. 3. FE model of a square laminate under uniaxial tension to achieve uniform strain.

Table 2. Comparison of measured and predicted strength for a laminate lay-up of $[0 / 45 /-45 / 90]_{\mathrm{s}}$

\begin{tabular}{|c|c|c|c|}
\hline \multirow{4}{*}{ Laminate lay-up -- $[0 / 45 /-45 / 90]_{\mathrm{s}}$} \\
\hline \multirow{2}{*}{ Off-axis angle } & \multicolumn{3}{|c|}{ Ultimate strength, MPa } \\
\cline { 2 - 4 } & Experiment [18] & Triplett [19] & Present \\
\hline 0 & 765 & 721 & 725 \\
\hline 7.5 & 752 & 731 & 749 \\
\hline 15 & 774 & 788 & 833 \\
\hline 22.5 & 832 & 885 & 987 \\
\hline
\end{tabular}

Table 3. Comparison of measured and predicted strength for a laminate lay-up of $[90 / 30 /-30]_{\mathrm{s}}$

\begin{tabular}{|c|c|c|c|}
\hline \multirow{4}{*}{ Off-axis angle } & \multicolumn{3}{|c|}{ Laminate lay-up -- [90/30/-30] } \\
\cline { 2 - 4 } & Experiment [18] & Triplett [19] & Present \\
\hline 0 & 966 & 1064 & 1086 \\
\hline 7.5 & 908 & 885 & 921 \\
\hline 15 & 837 & 782 & 823 \\
\hline 22.5 & 807 & 731 & 776 \\
\hline
\end{tabular}

Table 4. Comparison of measured and predicted strength for a laminate lay-up of [0/45/-30 $]_{\mathrm{s}}$

\begin{tabular}{|c|c|c|c|}
\hline \multicolumn{4}{|c|}{ Laminate lay-up -- [0/45/-30] } \\
\hline \multirow{2}{*}{ Off-axis angle } & \multicolumn{3}{|c|}{ Ultimate strength, MPa } \\
\cline { 2 - 4 } & Experiment [18] & Triplett [19] & Present \\
\hline 0 & 883 & 756 & 756 \\
\hline 7.5 & 843 & 769 & 780 \\
\hline 15 & 929 & 859 & 864 \\
\hline 22.5 & 1028 & 1026 & 1093 \\
\hline 26 & 1129 & 1167 & 1138 \\
\hline 30 & 1074 & 1026 & 1026 \\
\hline 45 & 818 & 744 & 804 \\
\hline
\end{tabular}

American Institute of Aeronautics and Astronautics 
Table 5. Comparison of measured and predicted strength for a laminate lay-up of [90/0/90/0 $]_{\mathrm{s}}$

\begin{tabular}{|c|c|c|c|}
\hline \multirow{3}{*}{ Off-axis angle } & \multicolumn{3}{|c|}{ Laminate lay-up -- [90/0/90/0] } \\
\cline { 2 - 4 } & Experiment [18] & Triplett [19] & Present \\
\hline 0 & 1126 & 1073 & 1086 \\
\hline 1.5 & 1140 & 1038 & 1087 \\
\hline 3 & 1074 & 808 & 1089 \\
\hline 4.5 & 1018 & 600 & 1093 \\
\hline 6 & 861 & 473 & 1099 \\
\hline 7.5 & 713 & 452 & 852 \\
\hline 15 & 394 & 346 & 441 \\
\hline 22.5 & 288 & 288 & 312 \\
\hline
\end{tabular}

\section{Strength of laminates with a hole under compression}

In this demonstration example a composite laminate with a hole under compression is considered and the present strength predictions are compared to the experimental strength measurements by Suemasu et al. [20]. The laminate is made of T800H/3633 Gr/Epoxy unidirectional plies; the ply-level properties are summarized in Table 6. The geometry and finite element model of the specimen are shown in Fig. 4. The width, length, and thickness of the laminate are denoted by $W=38 \mathrm{~mm}, L=118 \mathrm{~mm}$, and $t=2.2 \mathrm{~mm}$, respectively. The diameter of the hole located at the center is $d=6.35 \mathrm{~mm}$. The laminate lay-up is of $[-45 / 90 / 45 / 0]_{2 s}$, and it is made of T800H/3633 Gr/Epoxy.

As shown in Fig. 4, the specimen is clamped along the left edge and subjected to uniform end shortening of $\Delta$ via a rigid clamp along the right edge. Note that the rigid clamp only allows end shortening (horizontal displacement) along the right edge. The corresponding load, $P$, is then calculated as a reaction force in the FE analysis. The first ply failure and ultimate strength predictions from the present approach are compared with the experimental measurements by Suemasu et al. [20]. In Fig. 5, the present analysis prediction for the quasi-isotropic laminate layup is shown with dark solid line. In this figure, the circles represent the load at which the first-ply failure occurs; the squares denote the load at which deviation from the linear variation starts; and the triangles represent the ultimate load. It is observed that the predicted first ply failure is $16.2 \mathrm{kN}$ and the ultimate strength is $21.8 \mathrm{kN}$ as compared to $17 \mathrm{kN}$ and $23.2 \mathrm{kN}$ measured by Suemasu et al. [20], respectively. The agreement with the experiment is remarkably close.

In order to further demonstrate the effect of lamination on the strength, two other lay-ups of, $[90 / 0 / 90 / 0]_{2 s}$ and $[90 / 30 /-30 / 0]_{2 s}$ are also considered. The prediction for these layups are shown in Fig. 5 with blue and red dashed lines. As expected, these laminates have higher strength than the one tested by Suemasu et al. [20] as shown in Fig. 5.

Table 6. Ply-level properties for T800H/3633 Gr/Epoxy Suemasu et al. [20]

\begin{tabular}{|c|c|c|l|}
\hline$E_{11}$ & $148.0 \mathrm{GPa}$ & $X_{t}$ & $2000.0 \mathrm{MPa}$ \\
\hline$E_{22}$ & $9.56 \mathrm{GPa}$ & $X_{c}$ & $-1500.0 \mathrm{MPa}$ \\
\hline$G_{12}=G_{13}$ & $4.55 \mathrm{GPa}$ & $Y_{t}$ & \multirow{2}{*}{$50.0 \mathrm{MPa}$} \\
\hline$G_{23}$ & $3.17 \mathrm{GPa}$ & $Y_{c}$ & \multirow{2}{*}{$-150.0 \mathrm{MPa}$} \\
\cline { 1 - 2 }$v_{12}=v_{13}$ & 0.3 & $S_{12}$ & $100.0 \mathrm{MPa}$ \\
\hline$v_{23}$ & 0.49 & & \\
\hline$t_{p l y}$ & $0.1308 \mathrm{~mm}$ & & \\
\hline
\end{tabular}




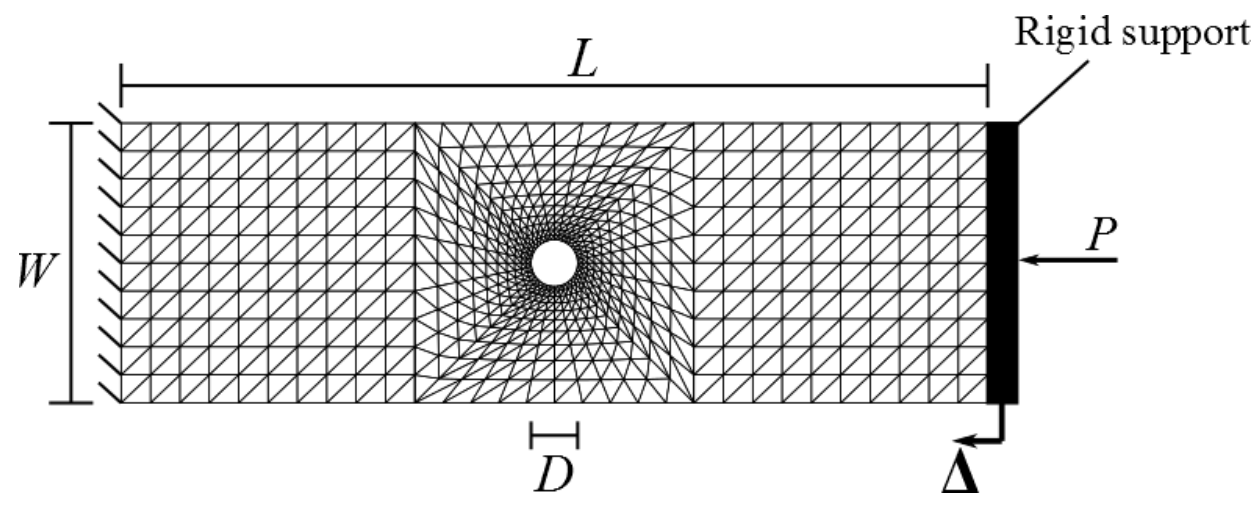

Fig. 4. Geometry, loading, boundary conditions, and finite element discretization for a compressively loaded laminate with a hole.

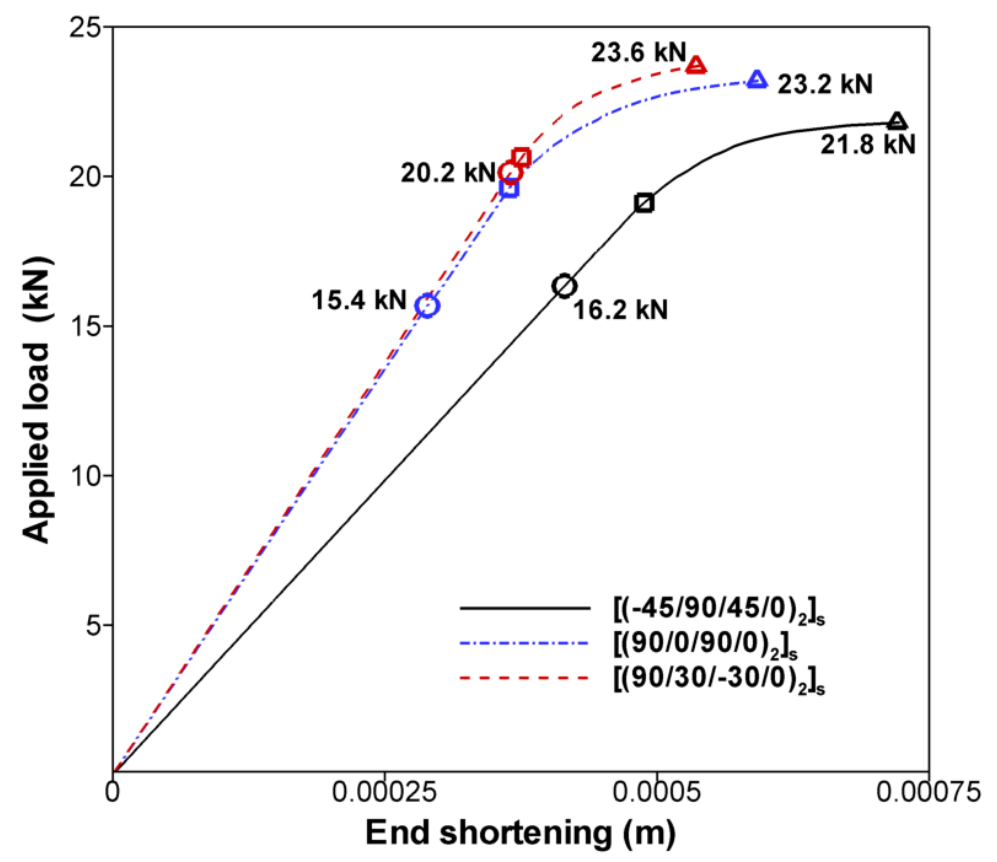

Fig. 5. Load vs. end-shortening response of compressively loaded laminates with a central hole

\section{Strength of laminates with a hole under tension}

In this example, a composite laminate with a hole under tension is considered, and the present strength predictions are compared with the experimental strength measurements by Chang and Chang [8] as well as previous predictions by Sleight [21], and Gunel and Kayral [22]. The geometry and finite element model of the specimen are shown in Fig. 6. The width, length, and thickness of the laminate are denoted by $W=25.4 \mathrm{~mm}$., $L=203.2 \mathrm{~mm}$., and $t=2.616 \mathrm{~mm}$., respectively. The diameter of the hole located at the center is $d=6.35 \mathrm{~mm}$. The laminate layup is made of T300/1034-C Gr/Epoxy unidirectional plies with a lay-up of $\left[0 /( \pm 45)_{3} / 90_{3}\right]_{s}$. The ply-level properties are given in Table 7.

While the side (horizontal) edges of the specimen are free from constraints, the loaded edge is subjected to uniform end-displacement of $\Delta$ via rigid clamps, and the left vertical edge is clamped as depicted in Fig. 6 . As shown in Fig. 7, the end shortening-load curve obtained from the present analysis for the laminate layup considered previously by others is represented with dark solid line. Similar to the previous example, the first ply, first deviation, and ultimate loads are, respectively, denoted by circles, squares, and triangles in Fig. 7. 
The first ply failure and ultimate load predictions from the present approach are compared with the experimental measurements by Chang and Chang [8] and previous predictions by Sleight [21] and Gunel and Kayral [22]. As shown in Fig. 7, the predicted first ply failure is $10.72 \mathrm{kN}$ and the ultimate strength is $16.7 \mathrm{kN}$ as compared to the measured ultimate strength of $15.6 \mathrm{kN}$ (experimental first ply failure is not available). The previous predictions by Sleight [21] and Gunel and Kayral [22] are, respectively, given as $(6.7 \mathrm{kN}, 14.3 \mathrm{kN})$ and $(8.0 \mathrm{kN}$, $16.5 \mathrm{kN})$, in which the first number in the parenthesis denotes the first ply failure load and the second number represents the ultimate load. It is observed that the comparisons against the experiment and other predictions indicate reasonably close agreement.

In order to further demonstrate the effect of lamination on the strength, two other lay-ups of, $\left[0_{3} /( \pm 45)_{2} / 90_{3}\right]_{s}$ and $\left[0_{3} /( \pm 45)_{3} / 90\right]_{s}$ are considered. In Fig. 7 , the results for these two laminate layups are represented by dashed red and blue lines, respectively. As expected, these laminates have higher stiffness than the one tested by Chang and Chang [8].

Table 7. Ply-level properties for of T300/1034-C Gr/Epoxy, Chang and Chang [8]

\begin{tabular}{|c|c|c|l|}
\hline$E_{11}$ & $146.85 \mathrm{GPa}$ & $X_{t}$ & $1723.7 \mathrm{MPa}$ \\
\hline$E_{22}$ & $11.4 \mathrm{GPa}$ & $X_{c}$ & $-1378.9 \mathrm{MPa}$ \\
\hline$G_{12}=G_{13}$ & $6.2 \mathrm{GPa}$ & $Y_{t}$ & $66.5 \mathrm{MPa}$ \\
\hline$G_{23}$ & $2.18 \mathrm{GPa}$ & $Y_{c}$ & $-268.2 \mathrm{MPa}$ \\
\cline { 1 - 2 }$v_{12}=v_{13}$ & 0.3 & $S_{12}$ & $133.7 \mathrm{MPa}$ \\
\hline$v_{23}$ & 0.49 & & \\
\hline$t_{p l y}$ & $0.1308 \mathrm{~mm}$ & &
\end{tabular}

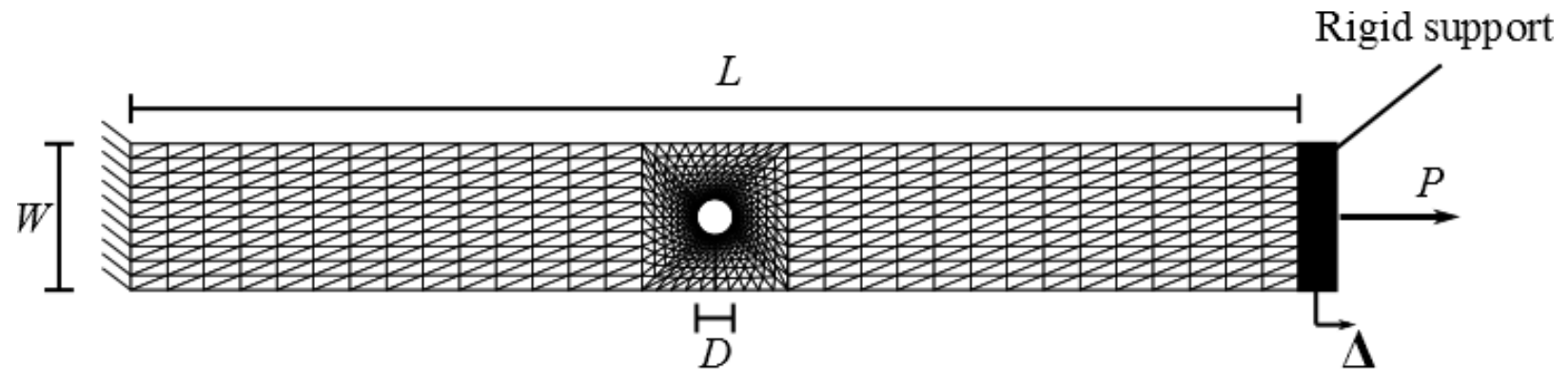

Fig. 6. Geometry, loading, boundary conditions, and finite element discretization for a laminate with a hole under tension. 


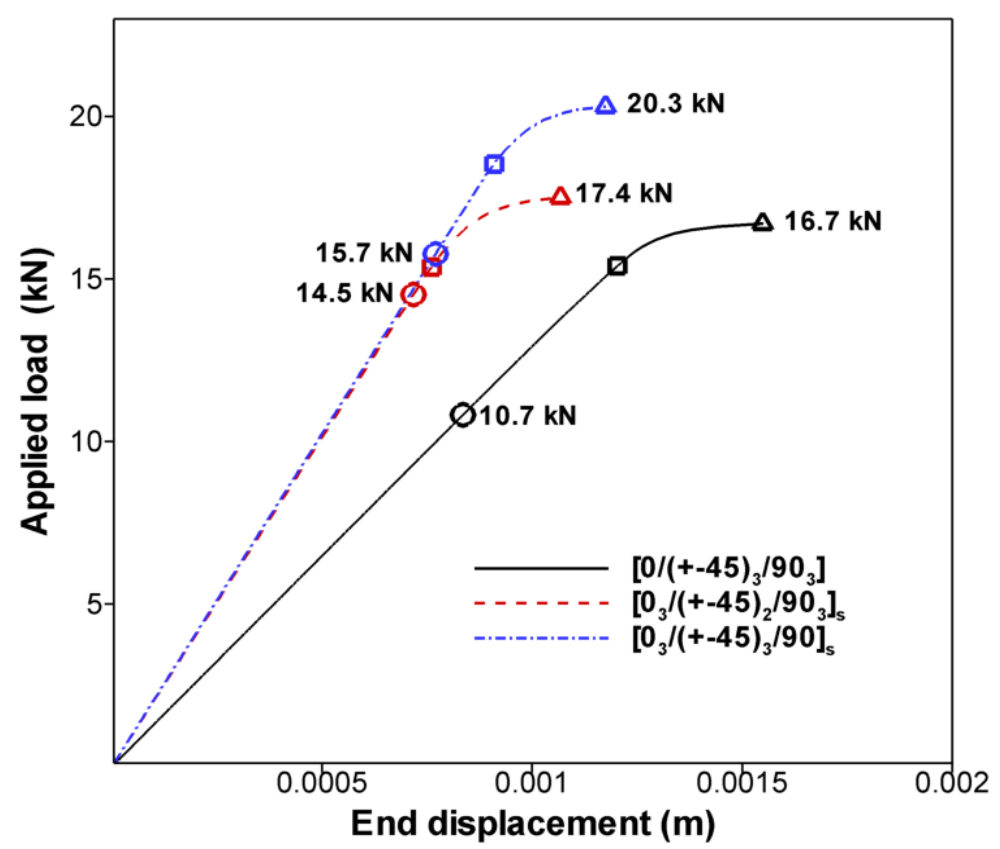

Fig. 7 Load vs. end shortening response of laminates with a central hole under tension

\section{Concluding remarks}

The refined zigzag element, $\mathrm{RZE}^{\{2,2\}}$, accounts for the discrete nature of fiber- and resin-rich layers of each ply as well as the stiffness degradation of any of the layers. This new element predicts accurate in-plane, transversenormal, and transverse-shear stresses; thus, it eliminates the lack of reliability associated with inaccurate computation of stress redistribution while performing progressive failure in composite laminates. This approach will enable the designers to consider various laminate lay-ups without relying on significant amount of material testing and characterization. As a result, significant reduction in cost and design cycle time are expected in the use of highperformance material systems as they become commercially available.

\section{References}

${ }^{1}$ Talreja, R.: Damage mechanics of composite materials, Elsevier Science Ltd, Oxford (1994).

${ }^{2}$ Lapczyk, I., Hurtado, J.A.: Progressive damage modeling in fiber-reinforced materials. Composites: Part A, 38, 2333-2341 (2007).

${ }^{3}$ Falzon, B.G., Apruzzese, P.: Numerical analysis of intralaminar failure mechanisms in composite structures. Part I: FE implementation, Composite Structures, 93, 1039-1046 (2011).

${ }^{4}$ Hashin, Z.: Failure criteria for unidirectional fiber composites. J Applied Mechanics, 47, 329-334 (1980).

${ }^{5}$ Sun, C.T.: Strength Analysis of unidirectional composites and laminates, Comprehensive composite materials. Elsevier Science, 1, 641-666 (2008).

${ }^{6}$ Ochoa O.O.and Reddy J.N., Finite element analysis of composite laminates. Kluwer Academic publishers, New York, (1992).

${ }^{7}$ Chang, F.K., Lessard, L. B.: Damage tolerance of laminated composite containing an open hole and subjected to compressive loadings: part I - analysis. J Compos Mater, 25, 2-43 (1991).

${ }^{8}$ Chang, F.K., Chang, H.-Y.: A progressive damage model for laminated composites containing stress concentrations. J Compos Mater, 21, 834-855 (1987).

${ }^{9}$ Seng, T.: A progressive failure model for composite laminates containing openings. J Composite Materials, 25, 556-577 (1991).

${ }^{10}$ Tessler, A., Di Sciuva, M. and Gherlone, M., A consistent refinement of first-order shear-deformation theory for laminated composite and sandwich plates using improved zigzag kinematics", J. of Mechanics of Materials and Structures, 5, pp. 341-367. (2010). 
${ }^{11}$ Tessler, A., Di Sciuva, M. and Gherlone, M., A refined zigzag beam theory for composite and sandwich beams", J. of Composite Materials, 43, pp. 1051-1081 (2009).

${ }^{12}$ Di Sciuva, M., Gherlone, M. and Tessler, A., A robust and consistent first-order zigzag theory for multilayered beams", In Advances in Mathematical Modelling and Experimental Methods for Materials and Structures: The Jacob Aboudi Volume (Eds. R. Gilat, L. Banks-Sills) Springer (USA), pp. 255-268 (2009).

${ }^{13}$ Tessler, A., Di Sciuva, M. and Gherlone, M., A homogeneous limit methodology and refinements of computationally efficient zigzag theory for homogeneous, laminated composite, and sandwich plates", Numerical Methods for Partial Differential Equations, 27, pp. 208-229 (2011).

${ }^{14}$ Gherlone, M., Tessler, A. and Di Sciuva, M. $\mathrm{C}^{0}$ beam elements based on the Refined Zigzag Theory for multilayered composite and sandwich laminates , Composite Structures, 93, pp. 2282-2294 (2011).

${ }^{15}$ Onate, E., Eijo, A. and Oller, S. Simple and accurate two-noded beam element for composite laminated beams using a refined zigzag theory, Computer Methods in Applied Mechanics and Engineering, 213-216, pp. 362-382 (2012).

${ }^{16}$ Versino, D. Gherlone, M., Mattone, M., Di Sciuva, M. and Tessler, A. $C^{0}$ triangular elements based on the Refined Zigzag Theory for multilayered composite and sandwich plates, Composites Part B: Engineering, 44B, pp. 218-230 (2013).

${ }^{17}$ Barut, A., Madenci, E., and Tessler, A., A refined zigzag theory for laminated composite and sandwich plates incorporating thickness stretch deformation, 53 ${ }^{\text {th }}$ AIAA/ASME/ASCE/AHS/ASC Structures, Structural Dynamics, and Materials Conference, Honolulu, Hawaii. (2012).

${ }^{18}$ Sun, C. T., Quinn, B. J., Tao, J., and Oplinger, D. W., "Comparative Evaluation of Failure Analysis Methods for Composite Laminates" DOT/FAA/AR.95/I09, (1996).

${ }^{19}$ Triplett, Matt H. "Progressive Failure Analysis of Fiber Composite Structures." Society of Plastics Engineers Annual Technical Conference, May (1999).

${ }^{20}$ Suemasu, H., Takahashi, H., Ishikawa, T.: On failure mechanisms of composite laminates with an open ole subjected to compressive load. Composite Science and Technology, 66, 634-641 (2006).

${ }^{21}$ Sleight D.W. Progressive failure analysis methodology for laminated composite structures, NASA/TP-1999209107.

${ }^{22}$ Günel, M. and Kayran, A., Linear and Non-linear Progressive Failure Analysis of Composite Aerospace Structures Under Combined Loading, 53 ${ }^{\text {th }}$ AIAA/ASME/ASCE/AHS/ASC Structures, Structural Dynamics, and Materials Conference, Honolulu, Hawaii., (2012).

${ }^{23}$ Knight, N. F. Jr., Factors Influencing Progressive Failure Analysis Predictions for Laminated Composite Structure," $49^{\text {th }}$ AIAA/ASME/ASCE/AHS/ASC Structures, Structural Dynamics, and Materials Conference, Schaumburg, IL, AIAA 2008-2108, (2008). 